\title{
Gallstone Ileus Presenting With Cholelith Emesis and an Incidental Benign Ovarian Fibroma: A Case Report
}

\author{
Paul Nderitu ${ }^{\mathrm{a}, \mathrm{b}}$, Anantole Wiik ${ }^{\mathrm{a}}$, Midhat Siddiqui ${ }^{\mathrm{a}}$, Imran Chishti ${ }^{\mathrm{a}}$
}

\begin{abstract}
Gallstone ileus (GI) is a rare but important cause of mechanical bowel obstruction associated with high levels of mortality. The classic radiological triad (Rigler's triad) of pneumobilia, bowel obstruction and an aberrant gallstone is present in $40-50 \%$ of cases. The vomiting of gallstones, "cholelith emesis", is a rare presentation of cholecystoduodenal fistula with bowel obstruction occurrence of which should prompt investigation for GI. Surgical management with enterolithotomy is the most common treatment option with fewer post-operative complications compared to one-stage procedures. We describe a case of GI in an elderly female patient presenting with cholelith emesis secondary to mid-ileal gallstone impaction and small bowel obstruction with an incidental large pelvic mass requiring emergency enterolithotomy.
\end{abstract}

Keywords: Gallstones; Ileus; Cholelith emesis

\section{Introduction}

Gallstone ileus (GI) is a rare cause of mechanical bowel obstruction occurring in $<0.1 \%$ of cases [1]. However, GI is associated with high levels of mortality (12-27\%), hence it should be treated as a surgical emergency [2]. GI is more common in elderly ( $\geq 65$ years) females and classically the radiological triad (Rigler's triad) of pneumobilia, bowel obstruction and an aberrant gallstone is present in $40-50 \%$ of cases [2]. We present a case of GI in an elderly female patient presenting unusually with cholelith emesis secondary to mid-ileal gallstone impaction and small bowel obstruction with an incidental large pelvic mass requiring emergency enterolithotomy.

\section{Case Report}

A 73-year-old female patient presented in clinic with a 1-year

Manuscript accepted for publication December 22, 2014

${ }^{a}$ Queen Elizabeth Hospital (NHS), Woolwich, London, UK

${ }^{b}$ Corresponding Author: Paul Nderitu, Queen Elizabeth Hospital (NHS), Woolwich, London, UK. Email: p.nderitu@doctors.org.uk

doi: http://dx.doi.org/10.14740/jcs258w history of irregular bowel habit but no weight loss, abdominal pain or significant medical or surgical history. Esophagogastroduodenoscopy and colonoscopy carried out 1 year prior showed she had a hiatus hernia, duodenitis and diverticular disease. Abdominal ultrasound performed 4 years earlier showed the presence of cholecystolithiasis. A computerized tomography of the chest, abdomen and pelvis (CT-CAP) was performed and showed evidence of gallbladder perforation with a $3 \mathrm{~cm}$ stone at the neck of the gallbladder and a $4 \times 3 \mathrm{~cm}$ intrahepatic abscess but no evidence of biliary dilation (Fig. 1); an incidental large cystic pelvic mass was also seen. Magnetic resonance imaging (MRI) of the pelvis revealed a large low signal pericystic mass posterior to the uterus measuring $16 \times 10.7 \times 14.6 \mathrm{~cm}$ suggestive of a pedunculated leiomyoma (Fig. 2). A drain for the liver abscess was placed whilst multidisciplinary team (MDT) discussion on further management was awaited.

Three weeks later, the patient represented with a 3-day history of nausea and bilious vomiting associated with decreased appetite. She also described vomiting numerous "stones" during this time. On examination, her abdomen was soft, nondistended but there was right upper quadrant tenderness and a mass in the right lower quadrant; bowel sounds and rectal examination were unremarkable as were her vital signs.

\section{Differential diagnosis}

Commonly, small bowel obstruction is caused by adhesions or hernia obstruction and where there is metabolic or electrolyte disturbance then pseudoobstruction should also be considered. Where there is evidence of adnominal pain, loose stool or weight loss, inflammatory bowel disease and neoplasms must be included in the differential diagnosis.

\section{Investigations}

Biochemical investigation revealed an elevated neutrophil count $\left(13.1 \times 10^{9}\right.$ cells/L), raised C-reactive protein (293 $\mathrm{mg} / \mathrm{L})$, creatinine $(177 \mu \mathrm{mol} / \mathrm{L})$ and urea $(9.6 \mathrm{mmol} / \mathrm{L})$; liver function tests, amylase and CA-125 were normal. MRI cholangiopancreatography found no evidence of biliary dilatation but showed multiple gallbladder stones. Repeat non-contrast 


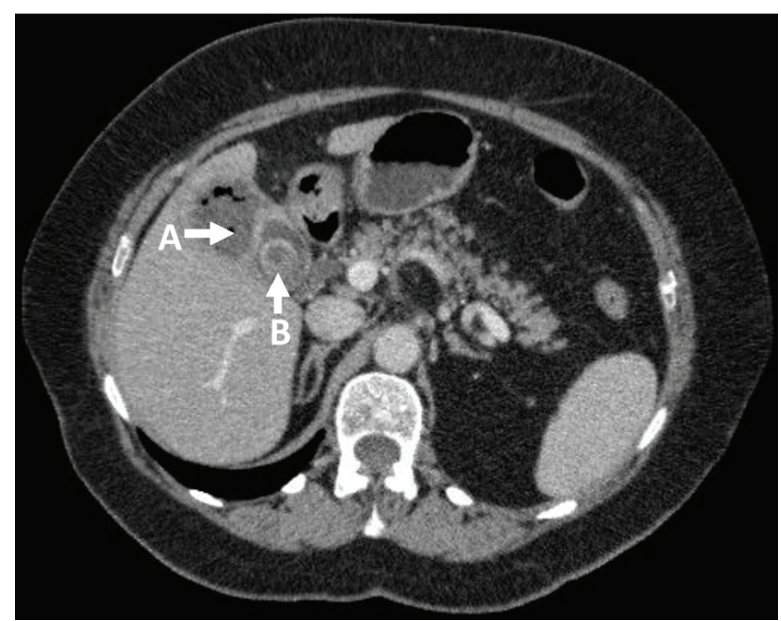

Figure 1. Axial CT-CAP: (A) liver abscess; (B) gallstone.

CT-CAP showed there was obvious small bowel dilatation, a $3 \mathrm{~cm}$ mid-ileal stone with concentric calcification (Fig. 3) and pneumobilia (Fig. 4) in keeping with cholecyto-enteric fistula; a large pelvic mass was also seen as well as small atrophic kidneys ( $9 \mathrm{~cm}$ bilaterally).

\section{Management}

The patient was initially treated conservatively with antibiotics but became increasingly symptomatic over the next 2 days. She therefore underwent an exploratory laparoscopy proceeding to laparotomy during which a large $(3 \mathrm{~cm})$ mid-ileal stone was seen (Fig. 5) as well as dilated small bowel loops. A large pelvic mass with associated fibrotic changes forming a point of constriction at the mid-ileum was evident (Fig. 6). Small bowel enterolithotomy and a right salphigo-oophorectomy were performed and tissue samples were sent for analysis. Histology showed the pelvic mass contained variable cellular

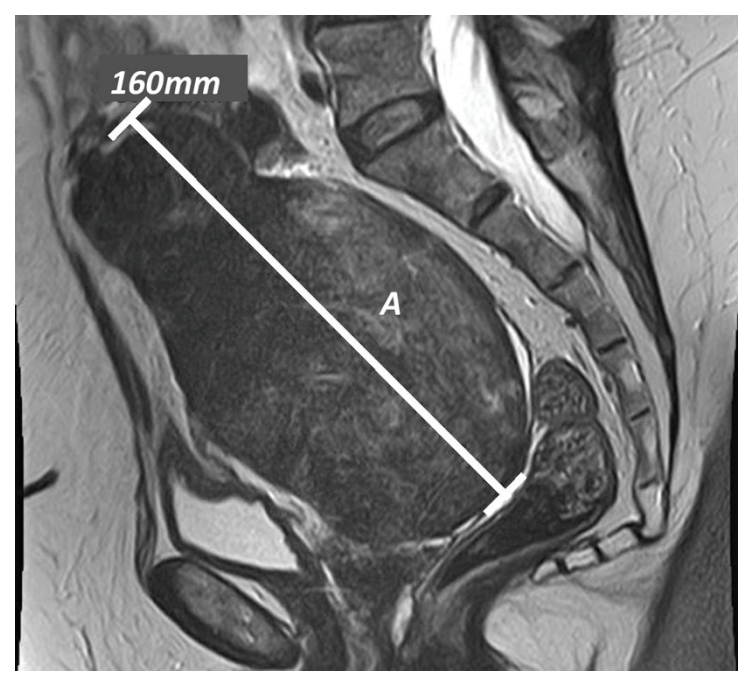

Figure 2. Sagittal T2 pelvic MRI: (A) pelvic mass.

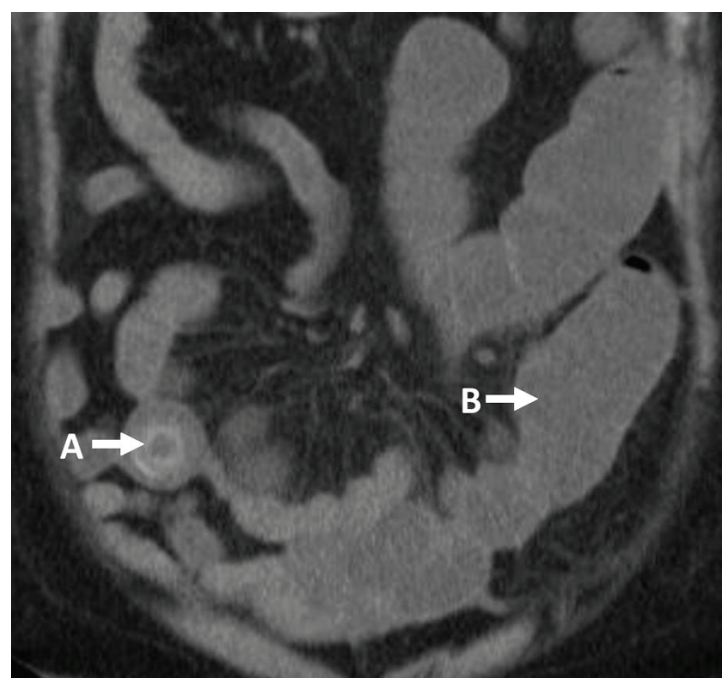

Figure 3. Coronal CT-CAP: (A) gallstone; (B) dilated small bowel loops.

proliferation without atypia, interlaced fibroblasts and cystic foci indicative of a benign ovarian fibroma.

\section{Outcome and follow-up}

The patient had an uncomplicated post-operative recovery and was discharged home after 7 days. She was seen in the surgical and gynecology outpatients 2 weeks later. There has been no recurrence of the ovarian mass and she is to undergo definitive elective cholecystectomy with fistulae repair.

\section{Discussion}

The first case of GI was described by Bartolini in 1654 [3]. GI is a complication of gallstones in $1-4 \%$ of cases and is more common in elderly females [2]. Symptoms at presentation are often non-specific as are biochemical investigations, although a raised white cell count and deranged urea and electrolytes

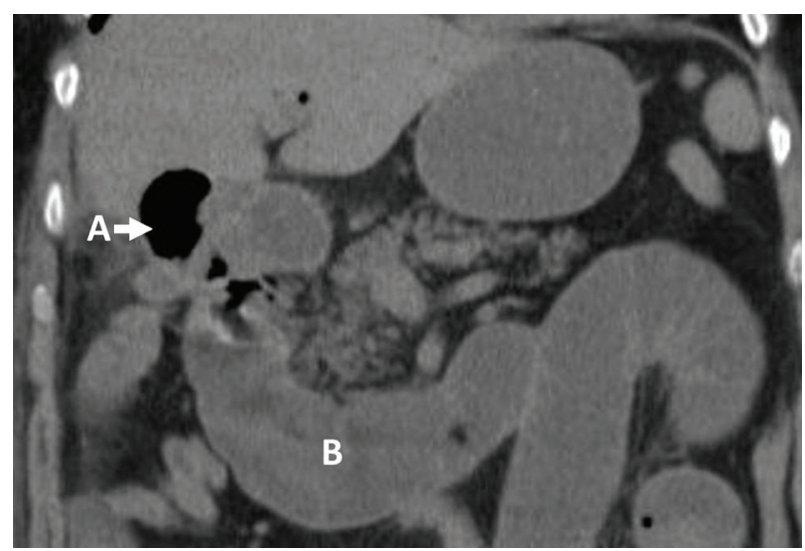

Figure 4. Coronal CT-CAP: (A) pneumobilia; (B) dilated small bowel loops. 


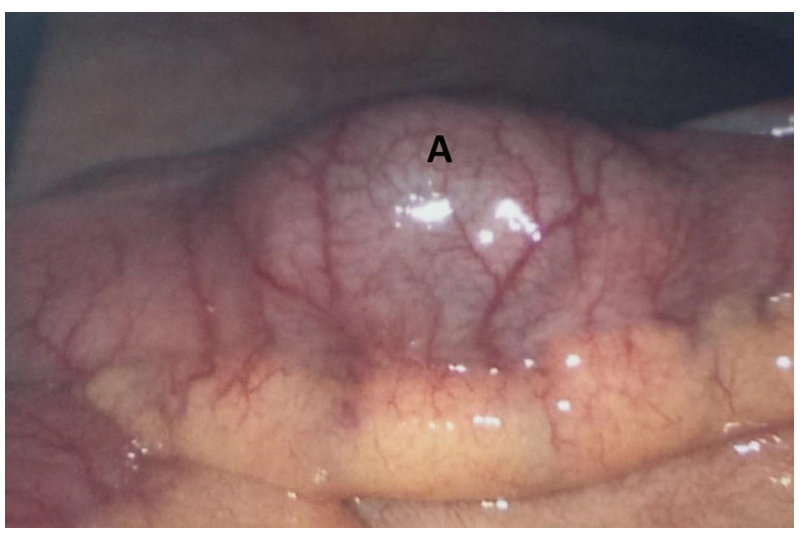

Figure 5. Diagnostic laparoscopy: (A) $3 \mathrm{~cm}$ aberrant gallstone in the mid-ileum.

are common [2]. Bilious vomiting is indicative of proximal obstruction [2] but vomiting of gallstones, "cholelith emesis", is highly unusual and itself is an indicator of bowel obstruction $[4,5]$. Clinical suspicion however remains key to diagnosis and GI should be considered in elderly patients with bowel obstruction, a history of cholelithiasis or presence of cholelith emesis [1].

Gallstones are normally $\geq 2.5 \mathrm{~cm}$ in diameter if there are to cause obstruction with smaller stones likely to pass spontaneously [2]. Fistulae formation with the duodenum is most often the route to the gastrointestinal tract but fistulization can occur with the colon, stomach or hepatic duct [3].

$\mathrm{CT}$ is the imaging modality of choice and the triad of pneumobilia, small bowel obstruction and an aberrant gallstone is present in $40-50 \%$ of cases [2]. However, in $50 \%$ of cases, the diagnosis is made during the operative procedure [2]. In $50-75 \%$ of cases, the gallstone impacts at the terminal ileum, $20-40 \%$ impact the jejunum or proximal ileum and $10 \%$ in the duodenum (Bouveret's syndrome) [2]. Unusually in this case, the patient had a large benign ovarian mass leading to small bowel adhesions and narrowing at the mid-ileum hence stone impaction occurred at this now narrow point leading to obstruction.

Laparoscopy alone occurs in a minority of cases $(10 \%)$ and $>50 \%$ of cases are converted to a laparotomy [3]. Enterolithotomy alone is performed in $80 \%$ of GI cases, $11 \%$ have a one-stage procedure with concomitant cholecystectomy/ otomy and fistulae removal; $4 \%$ of patients undergo other procedures and 5\% have no surgical intervention $[3,6]$. Patients undergoing enterolithotomy alone have significantly lower post-operative complications $(27 \%)$ compared to patients undergoing a one-stage procedure (61\%) [6]. Associated mortality is also lower for enterolithotomy (11.7\%) compared to a one-stage procedure (16.9\%) [6]. Spontaneous fistulae closure occurs in $>50 \%$ of cases but $5 \%$ of patients continue to have biliary symptoms [1].

In conclusion, GI is a rare but serious cause of bowel obstruction more commonly in elderly female patients. Clinical suspicion and prompt CT imaging are key to making the diagnosis but the vomiting of gallstones is a diagnostic marker of

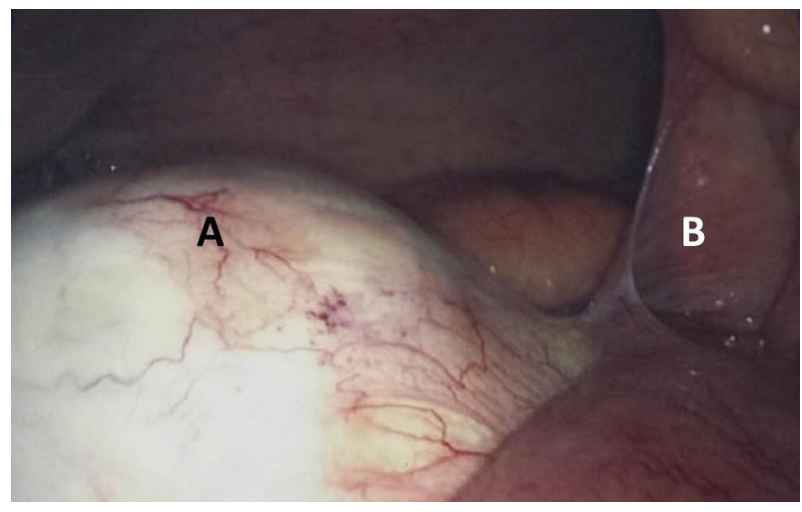

Figure 6. Diagnostic laparoscopy: (A) pelvic mass; (B) small bowel adhesions.

GI with bowel obstruction. Enterolithotomy is the most common surgical procedure performed in GI and is associated with a lower incidence of post-operative complications and mortality compared to a one-stage procedure. However, follow-up cholecystectomy and fistulae removal may be required in a second-stage procedure.

\section{Learning points}

1) GI is a rare but serious cause of mechanical bowel obstruction.

2) Cholelith emesis should prompt investigation for possible cholecystoduodenal fistula with bowel obstruction.

3) Radiological findings of pneumobilia, bowel obstruction and an aberrant gallstone are indicative of GI.

4) Enterolithotomy is associated with fewer post-operative complications.

\section{References}

1. Dai XZ, Li GQ, Zhang F, Wang XH, Zhang CY. Gallstone ileus: case report and literature review. World J Gastroenterol. 2013;19(33):5586-5589.

2. Reisner RM, Cohen JR. Gallstone ileus: a review of 1001 reported cases. Am Surg. 1994;60(6):441-446.

3. Halabi WJ, Kang CY, Ketana N, Lafaro KJ, Nguyen VQ, Stamos MJ, Imagawa DK, et al. Surgery for gallstone ileus: a nationwide comparison of trends and outcomes. Ann Surg. 2014;259(2):329-335.

4. Taylor PJ, Limbacher HP. The vomiting of gallstones. Report of a case. Am J Dig Dis. 1970;15(1):73-78.

5. Thomson WL, Miranda S, Reddy A. An unusual presentation of cholecystoduodenal fistula: vomiting of gallstones. BMJ Case Rep. 2012;2012

6. Doko M, Zovak M, Kopljar M, Glavan E, Ljubicic N, Hochstadter H. Comparison of surgical treatments of gallstone ileus: preliminary report. World J Surg. 2003;27(4):400-404. 